\title{
REPETICIÓN DE LA CULTURA SOCIALIZANTE DEL TRABAJO Y SUS REPERCUSIONES EN NICARAGUA
}

\begin{abstract}
Resumen
Se hace un recuento de la cultura del trabajo como algo fundamental de la vida humana la cual se construye socialmente. En ese sentido, se hace un recorrido de los aspectos más importantes desde el punto de vista de las experiencias latinoamericana en la construcción de prácticas culturales, las cuales son reflejos de un legado y una interacción de resistencias ante modelos ideológicos hegemónicos.
\end{abstract}

Palabras claves: cultura del trabajo, ideología, repetición, neoliberalismo, dependencia, modelo empresarial

Key words: work culture, ideology, repetition, neoliberalism, dependency, business model

\section{Introducción}

La cultura es una construcción humana, compartida y reproducida socialmente Estamos hablando de su condición dinámica, de su permanente movimiento y modificación (C. Marx). Todas las actividades humanas, codificadas y representadas, en concordancia con la variabilidad de cada cultura y entre estas, el trabajo, es una actividad fundamental en la vida de los seres humanos. Muchos pensamos que es correcta la afirmación de los antropólogos, en el sentido que esa validez es reconocible en todas las culturas, debido a su carácter estructural, material, evolutivo, histórico y biológico, al mismo tiempo que simbólico y espiritual. En suma, su vinculación con la vida social de los seres humanos. Y se conoce en la teoría antropológica y en general en las disciplinas sociales, como "cultura del trabajo".

Es un reclamo insistente de los teóricos de las ciencias sociales, en constantes debates, la necesidad de investigar los factores que condicionan o determinan sus modificaciones y cómo se produce y reproduce esta cultura del trabajo.

\section{Ideología y cultura}

Entendiendo la ideología en su acepción más amplia, (no solo política), sino como fuerza selectiva y emotiva de los materiales culturales a combinar en la construcción permanente de las culturas, o sea en su reproducción, coincidiendo con G. Gómez Pérez (1985). Aquella que la cultura latinoamericana, y dentro de ella, la cultura nicaragüense del trabajo, adquirió sus propias características,

Licenciado en Antropología, docente del Departamento de antropología. UNAN-Managua, Este artículo se publica como homenaje a su memoria. 
obedeciendo a los valores de esa ética social, en otras palabras, calzando sus valores con el resto de la cultura, que intuitiva o más o menos conscientemente, rescata ideológicamente de su concepción tradicional entrañablemente étnica o"el inconsciente colectivo" que nos analiza Aldo Díaz Lacayo (2007):

Pues bien, la historia se desarrolla a nivel consciente -tanto más consciente cuanto más difícil-, los mitos y la cosmogonía permanecen inalterables, sumidos en lo más profundo de la consciencia colectivasiempre difusos, presentidos, inconscientes, desarrollándose así el inconsciente colectivo de cada nación. Y aunque en la mayoría de los casos los individuos no se percaten de ello, es el inconsciente colectivo el que orienta e ilumina las acciones históricas de cada pueblo".

Es lo que hace poner en vigencia histórica en la práctica, también cultural, las contradicciones de esos inconscientes que los más se sientan, (figurativamente): herederos de indios pinoleros y otros de españoles toreros y gringos vaqueros.

En el título del artículo, hablamos de repetición. Es un término que fue introducido por el antropólogo Carlos Delgado O (1980), cuando se planteó la dependencia del imperialismo norteamericano, su imitación y traducción, por parte de los "sectores sociales intermedios", en procesos de arribismo en las sociedades de los países latinoamericanos. Según estos, los países subdesarrollados, dependen del desarrollo de los llamados desarrollados, antes del advenimiento de lo que podríamos llamar, la nueva etapa imperialista conocida como globalización que, a nivel mundial se produce como consecuencia de la derrota de la Unión Soviética en la guerra fría.
Para Latinoamérica, esa nueva etapa imperialista había empezado mucho antes, después de la independencia de España y Portugal. Los intentos sistemáticos por trazar la condición global surgieron hasta los años sesenta a través del desarrollo de conceptos como primero/segundo/tercer mundos, Norte/Sur, desarrollo/subdesarrollo, centro/ periferia, si bien desde principios del presente siglo encontramos reconocimientos de que la humanidad se estaba convirtiendo en una sola sociedad (hobhouse 1906). Agrega Ana Rosas M: Fue en el ámbito de las empresas transnacionales donde comenzó a emplearse el término globalización.

A finales de los ochenta e inicios de los noventa, el imperialismo capitalista inaugura su nuevo modelo construido con los restos y piezas del anterior, se hace llamar neoliberalismo, que es la misma expresión política liberal del capitalismo, pero exacerbada por la falta de contrapeso mundial equiparable. Dice F. Hinkelammert (1991):

"La crisis del socialismo ha debilitado extremadamente al tercer mundo que se encuentra totalmente solo frente al primer mundo: Un capitalismo que trató de aparecer durante las décadas de los cincuenta hasta los setenta como un capitalismo con rostro humano, ya no necesita hacerlo. El capitalismo de los cincuenta y sesenta, fue un capitalismo de reformas económicas y sociales que incluso se preocupó del desarrollo de países del tercer mundo, para no dejar chance a posibles movimientos alternativos. Pero ese capitalismo cree saber hoy que no existe ninguna alternativa se haga lo que se haga. Se puede ahora presentar como un capitalismo sin rostro humano. El capitalismo se siente en la situación de "hemos ganado" y propone un futuro en el cual ya no hay historia ni conflictos esenciales, en el cual el primer mundo ha 
encontrado su paz y el tercer mundo ya no cuenta. Un solo imperio engloba el mundo entero. Está en todas partes. Llega a tener el poder total y lo sabe. La autoproclamada sociedad abierta, constituye la primera sociedad cerrada en la que no existe ningún escape hacia afuera".

Desde la conquista y colonización europea, a lo que erróneamente llamaron, las Indias Orientales, el posterior dominio hegemónico por parte de Norte América y Europa, denominado imperialismo y neocolonial el tipo de dominación, para ponerle nombre nuevo a un nuevo tipo de colonización no declarada y luego, la globalización. Los pueblos de los Países latinoamericanos viven amenazados $\mathrm{o}$ sometidos al poderío económico y militar de esas potencias.

En la época colonial, los países europeos se disputaban violentamente el dominio de ese continente, compitiendo y arrebatándose entre ellas la acumulación de riquezas que garantizara su capital inicial y evitara su sometimiento. El comercio entre países creció tanto que presionó para el desarrollo de la Industria. Los viajes de exploración por el mundo y sus océanos, fueron impulsados por esa competencia de dominio y. conquista.

La acumulación de riquezas provenientes de la invasión a las nuevas colonias en siglo XVI, le dan un origen gigante al capital que simultáneamente va desarrollando la industria que el comercio internacional necesita y nuevos inventos para su crecimiento acelerado se concentra en las nuevas ciudades o burgos. Se crea una gran necesidad de trabajadores con mayor prole para la reproducción de las nuevas industrias y la infraestructura urbana, redes de transporte de todo tipo, instalaciones y comunicación más rápida, etc.
Luego de su independencia, los Estados Unidos, continuaron lo que habían empezado sus colonizadores, con el exterminio de los indios y expropiación de sus bienes. , A éstos los consideraban como raza inferior y su cultura natural e incivilizada, según su cosmovisión bíblica, uniéndose a los europeos en esa carrera.

Los pueblos sometidos desde el inicio de la invasión extranjera, fueron forzados a abandonar todas las partes de la cultura vinculadas a su gobierno y soberanía, cosmovisión y filosofía. Pero hicieron todo lo posible por conservar, inclusive en secreto o clandestinamente, lo más sagrado que pudieron, como el amor e imitación a la naturaleza y a la "madre tierra"; los espíritus de la naturaleza, la propiedad colectiva o social y el trabajo común; la familia extensa, la reciprocidad y ayuda mutua, todo entrelazado como una sola sabiduría. Además de todo aquello de naturaleza indígena, que no estuviera expresamente prohibido, valiéndose de todo tipo de artimañas y camuflajes para ese fin, pero acostumbrándose a asimilar lo impuesto y a acomodarlo de manera que no arrancara de raíz su sabiduría, en la medida de lo posible.

Otra característica de la dependencia, es que los centros de poder o metrópolis legalmente dispusieron que las colonias compren lo que producía Europa; y solo produzcan lo que los colonizadores necesitan, abastecerse si fuese posible sin pagar, lo que se llamó después, "términos desiguales de intercambio". Característica fundamental de la dominación que, con distintos nombres perdura hasta hoy. Aunque se hayan visto forzados a proponer procesos de autonomía industrial, con nombres como "sustitución de importaciones", "Alianza para el progreso", Consenso de Washington, Tratados de libre comercio, etc. 
La esperanza en una nueva vida libre de dominación extranjera nunca se perdió. La independencia, basada en alianzas de nativos, esclavos negros, mestizos y descendientes de los blancos invasores, era el murmullo general. La revolución liberal se encarnaba en la independencia de los Estados Unidos, la Revolución francesa y las reformas democráticas de Inglaterra y otros lugares de Europa.

Paralelamente, la industria europea se estaba afianzando. Era una fiebre revolucionaria liberal e independentista mundial. Se soñaba con superar todas las cadenas, segregaciones y abusos con la libertad individual y el contrato social. Una vida llena de nuevos inventos que parecían mostrar la evolución material y espiritual de todo. Se haría realidad el sueño de la felicidad. Nace el análisis de la esencia del estructural-funcionalismo que concibe la curación y restablecimiento del sistema vigente, retomando sus anhelos y saneando sus equivocaciones, pero que funciona y cada pieza funcional es necesaria y complementaria. Solo que no logran ver que el seguro de todo el andamiaje, es militar y policial, sin el cual, las propias contradicciones derrumbarían el sistema, como asegura el marxismo.

La independencia de la colonia española y portuguesa y europea en general, se produce en los países latinoamericanos, durante un modo de producción mixto, casi totalmente feudal, mercantil y agrario. Régimen de hacienda, mezclado con esclavismo, por ser constituida por indios y negros africanos. Eran unas clases pintadas de raza y etnia, nacidas del modelo empresarial de turno. Una pequeña industria privada y casi artesanal o artesanal con poca tecnología en la época de fiebre de descubrimientos e inventos que generaban esperanzas.
Con el devenir histórico, se afianza el capitalismo, introducido bajo la formación socioeconómica de enclaves y los centros de poder de los Estados Unidos e Inglaterra. Es cuando se disputa la dominación con Inglaterra y otros rivales europeos, la conocida Doctrina Monroe: América para los americanos.

\section{Trascendencia de la estructura de las relaciones sociales de producción y circulación en la estructura de toda la sociedad latinoamericana.}

El régimen empresarial impone unas relaciones de producción que sellan el estilo nuevo de la cultura del trabajo, reflejada en toda la vida social y cultural de cada época. Veamos lo que nos dice Darcy Ribeiro, 1972:

"Cada núcleo industrial surgió en estas áreas como un enclave aislado en medio de una economía arcaica preponderantemente, que solo le permitía expandirse cuando no se oponía a los intereses oligárquicos implicados en el latifundio y en la economía de exportación. Al estar todo el poder político monopolizado en manos de los sectores importadores y exportadores, que aspiraban únicamente a lograr una integración más lucrativa en el sistema mundial, no pudo surgir un empresariado moderno opuesto a la oligarquía. Por el contrario, esta misma se desdobló formando el empresariado industrial, asociándose a los intentos modernizadores promovidos por las corporaciones internacionales."

Las empresas capitalistas transnacionales del imperialismo: agrario, industrial, comercial o financiero, introdujeron en Nicaragua y en Latinoamérica, su estructura jerarquizada con funciones específicas, reflejada en su organigrama y 
tratándose de acomodar a las estructuras sociales precedentes. Jorge Jenkins (1978), se refiere al legendario héroe Augusto C. Sandino en los siguientes términos:

\section{"Antes de entrar a la vida militar. Sandino conoció personalmente las operaciones de empresas norteamericanas en territorio centroamericano y México. A su paso por Bluefields (1920), en donde permaneció durante un mes, tuvo la oportunidad de conocer una región estructurada de un modo muy diverso con respecto a la región del Pacífico, y en donde las empresas norteamericanas habian constituido una especie de república propia dentro de la misma Nicaragua. En el área de Bluefields, la United Fruit Company tenía el monopolio del banano, al menos desde 1889, con plantaciones que se extendian por los ríos Escondido, Rama, Siquia, Mico y Grande de Matagalpa. Bluefields era el sitio donde se concentraba la fruta para su exportación. La fuerza de trabajo estaba constituida principalmente por indios mískitos y creoles que ocupaban los puestos más bajos".}

Estas y otras referencias históricas, nos sirven para ilustrar la conclusión que, toda estructura o modelo empresarial transnacional dominante, con el tipo de dominación ejercido sobre Latinoamérica, que es a su vez expresión de su estructura productivo- económica, en las distintas épocas de la historia, influye determinante mente en la configuración de toda la sociedad dominada y en la selección ideológica de los elementos o componentes de su cultura; solamente en aquellos que garanticen su reproducción y garanticen su continuidad. Es aquí donde el prestigio-poder de los vencedores impone sus modelos, aunque no la reproduce totalmente, porque es contestado por la cultura tradicional de cada pueblo sometido, que se arraiga en sus tradiciones y resiste, según la trayectoria histórica de lucha, de éxitos y fracasos. Es ese inconsciente colectivo, al que se refiere Aldo Díaz Lacayo.

Hay que tener presente que para la producción económica- social, se requiere una cultura, cuyos protagonistas construyen, ideológicamente guiados por sus intereses de clase, normados por la estructura empresarial productiva y de servicios, desigualmente instaurada. Los acomodan a sus aparatos productivos, normas de funcionamiento material, herramientas, máquinas, plantas y hasta vestimenta. $\mathrm{Y}$ reglas jerarquizadas de manejo humano de esa producción, circulación y consumo.

¿Quienes mandan y quienes obedecen a quienes? Estas disposiciones llevadas a la práctica, son una camisa de fuerza para la reproducción de la normativa social, formalmente independentista. Se condiciona a sus desigualdades, dominaciones y libertades, encasilladas en tarifas de poder, emanado del patrimonio económico y control del régimen de ganancias de cada estamento, de este aparato productivo y abastecedor de necesidades materiales y espirituales; porque conllevan prestigio y deseos de imitación para ser aceptados y exitosos, y encima son bendecidos por los poderes religiosos de la época, que corean y conviven en el poder. Es por esto que Marx concluye, que no solo se debe estudiar y analizar la sociedad, sino también transformarla, porque a la hora de protagonizar la imposición de un sistema y su resistencia, se están construyendo las ideas que lo sustentan, en un proceso simultaneo, a la vez que continuado.

Para su reproducción, en la práctica, reacomodan su cultura, y lo hacen con entusiasmo de descubridores e inventores 
de cosas nuevas, aunque echen mano al inventario cultural histórico que está esperando para ser seleccionado y puesto en vigencia, siempre con preferencias o intereses, de la misma manera que un televidente selecciona los canales con un tele comando. Es por eso que las simbolizaciones de las identidades, no son precisamente auténticas, sino como podríamos llamar, prestadas, como las banderas políticas y muchos otros símbolos.

Luego el tiempo libre, aquel que puede considerarse descanso, reposición de fuerzas agotadas diariamente en el trabajo, distracción o fuga de la realidad o libertad de la mente, borradora de todas las opresiones y ultrajes. Ese tiempo libre, se debe compatibilizar con los valores de la producción, la propiedad y el poder y el privilegio o la marginación. Esa compatibilidad valorativa es decisiva en la reproducción de lo que se conoce como vida cotidiana.

Algunas falsificaciones de valores se instalan trasladándose del mercado, tales como ganador o perdedor, vivo o tonto; se asientan en la práctica, se les hace su carpintería, se norman o reglamentan y se nominan a sus custodios y responsables de su continuidad, se institucionalizan.

El estado, y su ordenamiento, pero fundamentalmente su represión, es la garantía de su reproducción. Es a esta dinámica a la que refieren los estructural-funcionalistas, prefiriendo la ignorancia de las causas que producen estos ordenamientos y su continuación reacomodada en el tiempo, debido a su empirismo y positivismo.

Es esta la fuerza de la estructura productiva que crea la cultura de imitación y repetición en gran parte de la sociedad, por el prestigio poder y se reproduce. Tiene su partida de nacimiento en los elementos que conforman las culturas producto de estas estructuras empresariales, son componentes comunes a toda la sociedad, a todas las clases e ideologías como el amor romántico, la profesionalización universitaria o las golosinas, el licor, las imitaciones de marcas privilegiadas. Eso es lo que buscan uniformizar los ingenieros de la superestructura cultural, para crear la imagen de que las clases ya no existen y mucho menos su lucha contradictoria, que toda la sociedad se puede llamar clase media, ya no son trabajadores, son clientes de las transnacionales y sus sucursales nacionales.

En Nicaragua y en Latinoamérica y también en otros continentes, surge el problema que las divisiones sociales, importadas en cada época; las clases que la empresa extranjera implanta y sus repetidoras nacionales remedan $y$ sus voceros televisivos, telenovelas, videojuegos, películas y la prensa (publicidad, farándula y propaganda), complementan traduciendo y repitiendo. Chocan con la característica distintiva de lo étnico que es la simbología de la liberación revolucionaria. Las etnias son el principal obstáculo para la reproducción fluida de esa cultura del trabajo en Latinoamérica y especialmente en Nicaragua y otros países, donde se están gestando procesos revolucionarios. Las clases no son del mismo material, son o provienen de distintos colores y raíces étnico-culturales. Por eso tantas masacres de pueblos indígenas en norte, centro y Sudamérica, en toda la historia de guerras internas (sobre todo) y limítrofes, con el fin acabar con lo que conceptúan la causa del atraso, hasta nuestros días.

Se originan identificaciones e identidades con historias de victorias y derrotas $\mathrm{y}$ resistencias heroicas y centenarias que 
respetar. Identidades de cada interés social que es la clave de los modelos revolucionarios en desarrollo. Ese recuerdo sometido pero orgulloso, anhelado para recuperarlo por su concepción de pasado glorioso, por su comunitarismo, el amor a la naturaleza y a la tierra como a una madre, justo cuando se descubren los resultados desastrosos de la desaparición acelerada de formas de vida de la tierra y su contaminación, el calentamiento global, todo por la maximización de ganancias del capitalismo global.

El modelo neoliberal, caracterizado por privatizar la empresa estatal, reducirla a su socio menor supervisor, traslada la estructura de la mesa directiva de la empresa privada, al mismo estado, la tiranía del accionista mayoritario.

En un primer momento, las organizaciones de trabajadores y pobres en general y el movimiento popular en el mundo retroceden, se produce una reconcentración de la riqueza aceleradamente, se recortan todos los derechos adquiridos y conquistados por los trabajadores; se impone la "nueva economía", se descentralizan las empresas y se retacean los sindicatos; el trabajo se hace precario, temporal, inestable, se congelan los salarios, se propicia la desocupación que desmoviliza a la organización de los trabajadores y siembra la esperanza de soluciones individualistas y competitivas. Se llega al extremo de la especulación financiera del capital, convertido en burbujas. Los empresarios capitalistas prácticamente hambrean a sus compradores, los trabajadores y como consecuencia a sus abastecedores de pequeñas y medianas empresas, en una palabra: crisis.

El entorno histórico dentro del cual se desarrolla la cultura del trabajo actualmente en Latinoamérica y el mundo, es fundamental., lo conforman los hechos y sus concepciones culturales socializadas y correspondientes, elegidas ideológicamente del inventario cultural histórico, de carácter económico, político, social, cultural, y étnico.

Es bajo el ordenamiento de la globalización que nos aproximamos a analizar la sociedad nicaragüense actual. Globalización que tiene varias caras del mismo fenómeno, es como todo fenómeno histórico, multifacético, estructural (y súper estructural al decir de C. Marx). Daniel Mato lo define así:

"El concepto de globalización en su carácter general se presenta con un carácter ambiguo, al admitir distintos contenidos. Ello es manifestación de que este proceso es una tendencia histórica resultante de diversos procesos sociales de alcance mundial, que apuntan hacia una sinergia global interconectando diferentes regiones y países, en virtud de múltiples y complejas interrelaciones, incluyendo no sólo el aspecto económico, sino también social, político, ideológico y cultural. Como tendencia este proceso produce interrelaciones de organizaciones sociales geográficamente distantes entre sí $e$ intensifica interconexiones preexistentes" (Daniel Mato, 1993, Caracas).

Revienta la crisis en las metrópolis, la parálisiseconómica, bajanlasimportaciones y exportaciones. Muchos de los medio pobres, pobres y no tan ricos, derechistas admiradores de la propaganda capitalista en los países pobres, se desilusionan de su costosamente entrenado futuro, dudan y eligen (ideológicamente), apoyar a los otros pobres y sus organizaciones llamados de izquierda o revolucionarios, por estar en oferta el "único palo en que ahorcarse". Aparecen gobiernos de Izquierda en 
Latinoamérica, ganando las elecciones y uniéndose entre ellos para proteger su desarrollo y para encontrar intercambios alternativos y compensatorios en el comercio y en otros campos de actividad cultural como la Alianza Bolivariana ALBA.

El modelo empresarial que modelaría la transformación de la estructura social en Nicaragua, es el modelo cooperativo, que fue incorporado por el héroe legendario, fundador de la revolución, Augusto C. Sandino en su temprana planificación de lo que sería la nueva sociedad. Conocedor de la trascendencia de la estructura empresarial productiva y de servicios en el ordenamiento de toda la sociedad, lo que hoy conocemos los antropólogos como cultura del trabajo y su caracterización socialista autogestionaria.

El Científico Social Orlando Núñez Soto, es uno de los que analiza y defiende el planteamiento de desarrollo autogestionario en Nicaragua y con perspectivas latinoamericanas. Postulado que coincide con la herencia de Sandino, las experiencias en distintas partes del mundo y las nuevas características que va adquiriendo el movimiento revolucionario, alimentado con la historia del comunitarismo indio pre-hispánico. Más aún, si consideramos que la pobreza facilita el refugio en la tradición, porque es compatible con la marginación económicosocial-cultural y es, por lo mismo, una barrera para el cambio globalizante.

La estructura social de las sociedades originarias, precolombinas, correspondía evidentemente a su propio modo de producción, pero en un entramado compatible entre economía (entendida como transformación de la naturaleza con trabajo para satisfacer necesidades humanas con responsabilidades sociales expresas) y cultura, difícil de separar aún con fines epistemológicos, por eso existe tanta polémica en torno a la importancia de cada categoría y concepto y su mutua vinculación. En esas sociedades, donde los jefes de estado o pueblo o los órganos colectivos de gobierno, eran al mismo tiempo sumo sacerdotes y los espíritus de la naturaleza eran parte de la producción.

La familia y el parentesco, el rito y la danza se correspondían con el sistema productivo y de distribución e intercambio, así como la organización social comunitaria y el trabajo colectivo, tal como es apreciado por Maurice Gogelier (1976) en sus trabajos y de otros en varios continentes. La cultura del trabajo de ese régimen de vida social era parte de todo ese entramado sociocultural y para estudiarlo y comprenderlo, se tiene que buscar una aproximación interdisciplinaria y compleja, que requiere de reestructurar las metodologías y acomodarlas ideológicamente a los fines que los investigadores persigamos como parte de los buscadores de soluciones socioculturales colectivas.

\section{Bibliografía y webgrafía.}

-Corominas Jorge y Ribas Judith, 1992, Pensamiento e Identidad Latinoamericana, Managua, Departamento de Filosofía, Universidad Centroamericana.

-Delgado Carlos, 1980, Problemas Sociales en el Perú Contemporáneo, I.E.P.Campodónico Edic. Lima.

-Díaz Lacayo Aldo, 2002, El inconsciente Colectivo, s/e. Nicaragua.

-García Canclini, Nestor. 1995, Consumidores y ciudadanos. Conflictos multiculturales de la globalización. Grijalbo, México. 
-Godelier Maurice,1976, Antropología y Economía, Editorial Anagrama, Barcelona.

-Gómez Pérez Germán, 1985, La Polémica en Ideología, F.C.E. México.

-González Arencibia, Mario. Globalización cultural una aproximación conceptual. Monografía.com en: http://www. monografias.com/trabajos $11 /$ revcult/ revcult.shtml.

-Hart Dávalos, Armando. Cultura para el desarrollo. El desafío del siglo XXI. Universidad de Antioquia, enero del 2004.

-Hinkelammert Franz, 1991, La transición Dificil, Democracia, estructura económico-social y formación de un Sentido Común Legitimador, Editorial Vanguardia, Managua.

16 -Jenkins Moliere Jorge, Los Miskitos, 1980, Ed. Nueva Nicaragua

-Marx, Carlos. "Futuros resultados de la dominación británica en la India". Londres julio de 1853. En: Marx y Engels. Acerca del colonialismo
(Artículos y Cartas). Editorial Progreso, Moscú 1983..

-Mato, Daniel. "Procesos culturales y transformaciones socio-políticas en América Latina en tiempos de globalización". En: América Latina en tiempos de globalización: procesos culturales y transformaciones sociopolíticas. (Editor Daniel Mato) Caracas, 1995.

-Núñez Soto Orlando, 2011, La Economía Social Solidaria en la Naciones Proletarizadas y el Proletariado Por Cuenta Propia en la Transformación del Sistema, CTCP-FNT, Managua.

-Ribeiro Darcy, 1972, Configuraciones, Sep-setentas México.

-Rosas Mantecón Ana, 1993, Globalización Cultural y Antropología, Alteridades 3 (5), México.

-Telesur. http://www.causapopular. com.ar/article317.html.

-Wallerstein Immanuel, 1991, The National and the Universal: can there be such a thing as world culture, Ed, King, New York. 